\title{
Activity-Based Protein Profiling of Microbes
}

Natalie C. Sadler \& Aaron T. Wright ${ }^{\star}$

Biological Sciences Division, Pacific Northwest National Laboratory, Richland WA 99352, USA

${ }^{*}$ Corresponding Author:

Aaron T. Wright

902 Battelle Blvd

Box 999, MSIN J4-02

Richland, WA 99352

$1-509-372-5920$

Author Emails:

Aaron.Wright@PNNL.gov

Natalie.Sadler@PNNL.gov 


\section{Abstract}

Activity-Based Protein Profiling (ABPP) in conjunction with multimodal characterization techniques has yielded impactful findings in microbiology, particularly in pathogen, bioenergy, drug discovery, and environmental research. Using small molecule chemical probes that react irreversibly with specific proteins or protein families in complex systems has provided insights in enzyme functions in central metabolic pathways, drug-protein interactions, and regulatory protein redox, for systems ranging from photoautotrophic cyanobacteria to mycobacteria, and combining live cell or cell extract ABPP with proteomics, molecular biology, modeling, and other techniques has greatly expanded our understanding of these systems. New opportunities for application of ABPP to microbial systems include: enhancing protein annotation, characterizing protein activities in myriad environments, and reveal signal transduction and regulatory mechanisms in microbial systems.

\section{Introduction}

Activity-based protein profiling (ABPP) is a powerful strategy for dissecting the function of microbial systems relevant to human health, bioenergy production, and the environment. Characterization of enzymes, transporters, protein localization, regulatory dynamics, and new drug discoveries has been accomplished using chemical probes with advanced proteomic techniques. ABPP encompasses the application of an increasingly broad collection of probe motifs that vary in both protein reactivity and mode of binding in diverse biological samples. Chemical probes have been intuitively applied to cell extracts and living systems with characterization by gel electrophoresis, fluorescence microscopy, and/or mass spectrometry. To date, ABPP in microbial research have only begun to be applied, but great promise remains in research such as uncovering the mechanisms by which energy-relevant microbes and communities and microbial pathogens function and respond to external stimuli. Here, we discuss the basis of ABPP and its application to microbial systems. 


\section{ABPP_Probe Design Strategies}

We broadly apply the term ABPP to a diverse collection of probes with multiple modes of reactivity in vitro and in living organisms (Table 1): (1) Mechanism-based probes report on function by reaction dependent upon the catalytic mechanism of the target enzymes; (2) Probes that mimic small-molecules/metabolites for identifying specific protein interactions, e.g., drugs or small molecule probes with electrophilic or photoreactive moieties; and, (3) Probes that report on protein regulatory modifications. Each probe type consists of three moieties: a reactive group that forms an irreversible covalent bond with a target protein, a binding or spacer group (e.g., protein substrate or metabolite) that directs a probe toward a protein or protein family and may also impart cell permeability, and a reporter tag for rapid and sensitive measurement of labeled enzymes by fluorescence or mass spectrometry-based proteomics (Figure 1). Probes are often designed "tag-free" with an alkyne or azide compatible with the bioorthogonal HuisgenSharpless [3+2] cycloaddition reaction ("click chemistry") for multimodal reporter tag attachment [1]. This also decreases probe size, thereby minimizing undesirable impacts on reactivity with target proteins, and increasing cell permeability [2-5].

\section{Activity-Based Protein Profiling with Antibiotic Derived Probes}

Activity-based strategies are well suited for characterizing the mode of action by which drugs inhibit or activate an enzyme, enzyme family, or metabolic pathway, and for unraveling noncanonical drug metabolism pathways. Several ABPP studies have focused on antibiotics and antibiotic resistance. In 2008, Staub et al. synthesized natural and synthetic beta-lactam probes for application in antibiotic resistant Bacillus licheniformis, Listeria welshimeri, and Pseudomonas putida [6]. The beta-lactam probes labeled and inhibited a subset of the penicillin binding proteins including the virulence-associated enzyme ClpP and a resistance associated beta-lactamase. These results indicate potential highly selective targets for fine-tuned antibiotic 
discovery. A similar ABPP approach was utilized to characterize the effects of the nucleoside antibiotic showdomycin. A tag-free showdomyocin probe was applied to Staphylococcus aureus and inhibited enzymes involved in cell wall biosynthesis, notably the essential MurA1 and MurA2 [7].

In 2011, Eirich et al. sought to profile the targets of the antibiotic vancomycin. Despite an established mode of action, questions remain about the drug. These questions prompted researchers to develop a series of vancomycin probes enabled with a photoreactive moiety for binding target proteins and were then employed to identify non-canonical vancomycin targets in live Staphylococcus aureus and Enterococcus faecalis. Two uncharacterized targets were identified with likely roles in vancomycin's efficacy: autolysin in S.aureus and an ABC transporter in E. faecalis [8]. In a similar approach, derivatives of the $\beta$-lactam-containing antibiotic cephalosporin were used to identify active penicillin-binding proteins (PBPs) in both Bacillus subtilis PY79 and an unencapsulated derivative of D39 Streptococcus pneumonia. The probes were applied in vivo to explore cell wall PBPs during cell division. Fluorescence microscopy indicated cephalosporin C-based probes as more selective than a penicillin $\mathrm{V}$ analogue, which labels all PBPs [9]. Similar approaches may lead to rational design of drug variants with improved selectivity and potency, and for identifying potential routes of microbial drug resistance.

Recently, an ABPP approach was employed with 4-hydroxyderricin, an isolate from the plant Angelica keiskei which features antibiotic potency against several strains of bacteria including Staphylococcus aureus. The 4-hydroxyderricin probe bound and inhibited seryl-tRNA synthetases (STS), enzymes crucial for the biosynthesis of proteins. Sample alkylating pretreatments resulted in reduced probe labeling suggesting that the probe was binding to one or more Cys residues in the protein. Mutation studies were then performed to selectively replace each of the five cysteines with alanine; interestingly all five mutant enzymes displayed no 
catalytic activity emphasizing that a perturbation of just one Cys is sufficient to interfere with the amino acylation of tRNAs by STS. The results suggest that 4-hydroxyderricin inhibits STS activity by the alkylation of essential Cys residues within the enzyme and may induce apoptosis by halting protein biosynthesis. These results point towards a previously unrecognized role in regulation by this enzyme and illuminate it as a novel drug target [10]*.

\section{Activity-Based Protein Profiling of Enzyme Function in Microbial Pathogens}

\section{Serine Proteases}

Serine proteases are a widespread group of proteolytic enzymes critical to numerous physiological processes and implicated in microbial virulence mechanisms. Several probes to broadly characterize the serine hydrolase family of enzymes via a fluorophosphonate (FP) reactive group have been developed following initial work by Cravatt and coworkers [11]. While these probes are widely employed in mammalian systems, microbial research has lagged. Using a FP-probe multiplexed with a probe that targets enzymes with reactive cysteines in the fungus Aspergillus fumigatus, a human pathogen that is difficult to detect and for which no known virulence proteins are known, our group found that in the presence of host serum the organism alters physiological functions throughout numerous metabolic processes [12]. A follow-up study comparing $A$. fumigatus to two highly related but non-pathogenic fungi further confirmed the metabolic attenuation of $A$. fumigatus in the presence of the host, and the disparate metabolism in the presence of host was not identified in the other fungi [13]. Thereby, ABPP facilitated an improved understanding of the fungi's pathogenesis mechanism, and an array of proteins was identified as potential disease candidate biomarkers.

In a bacterial study, FP-probes detected the catalytic integrity of active site residues of the Rhomboid protein GlpG, a highly conserved intramembrane serine protease, in E. coli. 
Residues were identified located on the cytoplasmic side of the lipid bilayer required for maximal proteolytic activity, highlighting the utility of ABPP in characterizing enzyme function [14].

\section{Kinases/ATPases}

Protein kinases and ATPases phosphorylate a wide range of proteins by hydrolysis of ATP and transfer of a single phosphate moiety, a critical mechanism of intracellular signaling. Commencing with work by Patricelli, researchers have created probes as ATP mimics that selectively label active kinases and other ATPases in native proteomes [15]. The limited functional annotation for Mycobacterium tuberculosis is a common problem in microbial research that can be addressed using ABPP. To better characterize the biology of $M$. tuberculosis, and in pursuit of novel drug targets, Ansong et. al. created a tag-free ATP probe resulting in the characterization of $\sim 325$ ATPases, including 72 previously identified as hypothetical or unknown [16]. Many of the identified proteins are both unique to Mycobacteria and essential for infection, suggesting specialized functions in mycobacterial physiology and pathogenicity. Two subsequent studies evaluated ATPase function during latency of the pathogen. Oxygen is an environmental cue that triggers $M$. tuberculosis to be either in a latent or active state, but the mechanism used to regulate this oxygen-dependent replication switch is undetermined. Wolf et. al. characterized ATPases in M. tuberculosis in both regular and hypoxic growth conditions, finding differential function in several proteins involved in energy metabolism and lipid biosynthesis in hypoxic versus normal growth [17]. The second study also evaluated aerated versus hypoxic growth and determined that kinase inhibition led to a bacterial survival defect and that specifically, the PknB kinase is a critical regulator of the oxygen-dependent replication switch and is a promising drug target $[18]^{\star *}$.

In more targeted quantitative proteomic studies, a hypothemycin derived probe was developed to interrogate trypanosome Cys-Asp-Xaa-Gly motif (CDXG) kinases when coupled 
with RNA interference strategies. The two kinases eliciting the greatest reduction in probe labeling after exposure to hypothemycin, TbGSK3short and TbCLK1, are also the only CDXG kinases whose knockdown by RNAi significantly reduced cell growth [19]. Combined, these results provide strong evidence that these kinases are integrally involved in $T$. brucei infection mechanisms, and demonstrate the value of ABPP for drug/inhibitor target selectivity and potency.

High-throughput methods with ABPP have also been used in bacterial kinase studies to identify new antibiotics that reduce streptokinase transcription without inhibiting bacterial growth. Streptokinase is a bacterial activator of human plasmin and enhances Group A Streptococcus (GAS) virulence. From the screening seven compounds were identified as inhibitors, and three were developed into tag-free photoreactive probes for subsequent determination of their cellular targets [20]. This research is still in the preliminary stages of biological testing, but the overall strategy from inhibitor identification, to probe development, and chemoproteomic target identification is highly promising for developing new antibiotics in the future.

\section{Fatty Acid Synthases}

Intriguing research ventured into the realm of in vitro and in vivo labeling of native acyl carrier proteins (ACPs) in both type I and II fatty acid synthases. Pantetheinyl-probes were applied to bacteria and found to cross the cell membrane before transformation intracellularly into CoA analogues in vivo by the endogenous CoA biosynthetic pathway. These studies successfully used the probes to enrich carrier proteins and allowed for the visualization and proteomic identification of fatty acid ACPs in E. coli, B. subtilis, S. oneidensis, and B. brevis lysates [21-23].

\section{Glycoside Hydrolases}


Renewable energy research has focused on the degradation and fermentation of lignocellulosic biomass and non-food crop fibers to produce biofuels. Historically, identifying functional enzymes responsible for biomass degradation is very challenging. A suite of probes based upon aglycone, N-halogenated glycosylamine, and 2-deoxy-2-fluoroglycoside scaffolds were developed for labeling cellulases, hemicellulases, xylanases, and other glycoside hydrolases [24]* . The suite was applied to the highly efficient lignocellolytic anaerobic bacterium Clostridium thermocellum, and proteomics provided a picture of the functional assembly of the cellulosome, a cell-adherent cellulolytic assemblage of proteins. A subsequent study designed to facilitate biorefinery and biotechnology applications of glycoside hydrolases used the same probes to characterize the functional response of enzymes to mild and significant perturbations in temperature, $\mathrm{pH}$, and culture duration in the fungus Trichoderma reesei [25]. The suite of probes for glycoside hydrolases provides opportunities for optimizing biofuel processing conditions, and identifying enzymes for incorporation into enzyme "cocktails" for potentially improving biofuel production.

\section{ABPP to Characterize Protein Redox Dynamics}

Reduction and oxidation (redox) is a common post-translational protein modification playing myriad rolls in biological systems, including significant and often elusive roles in signaling and regulation. Cysteine thiol alkylating reagents such as iodoacetamide and $\mathrm{N}$ ethylmaleimide have been used to quantitatively characterize redox reactive cysteines in biological systems, and can be applied in conjunction with isotopic labeling for highly precise quantitation $[26,27]$. To decipher proteins sensitive to $\mathrm{H}_{2} \mathrm{O}_{2}$ stress in Pseudomonas aeruginosa and Staphylococcus aureus, differing oxidation conditions and subsequent cysteine redox status were profiled using isotopic iodoacetamide probes with tandem proteolytic methods to identify probe labeled Cys residues [28]. Biochemical experiments and chemoproteomics elucidated highly sensitive Cys thiols, including the master quorum sensing regulator LasR, and redox- 
regulated activities for acetaldehyde dehydrogenase ExaC, arginine deiminase ArcA, and glyceraldehyde 3-phosphate dehydrogenase GAPDH [29] ${ }^{\star *}$.

A challenge in characterizing protein redox dynamics is that cell lysis results in rapid oxidation, largely destroying the native intracellular redox state. Previously, multiple artificial reduction and oxidation steps have been used to induce protein redox while alkylating free thiols between each step in cell lysates, or by performing acid quenching concomitant with cell lysis $[30,31]$. To capture live cell biological redox phenomena in the biofuel relevant cyanobacteria Synechococcus sp. PCC 7002, tag-free electrophilic N-ethylmaleimide and iodoacetamide derived probes were redesigned to include a polyethylene glycol region to enable cell permeability. Two studies were designed to induce a change in cellular redox, and provide insight into post-translational redox modifications of proteins in metabolic pathways pertinent to biofuel or high value added small molecules. The first study evaluated a transition in carbon availability, from C-limited to C-starved then to C-replenished, with in vivo probe labeling throughout a 60-minute evaluation of redox dynamics [32]. Dramatic changes in the redox state were characterized for 176 proteins within 30 seconds of carbon reintroduction by label-free quantitative proteomics, with subsequent identification of probe-labeled Cys residues using the tandem protease cleavage method previously described $[28,29]^{\star *}$. This study employed highresolution confocal fluorescence microscopy and protein identification and LC-MS/MS-based proteomics approaches [32]. A second study profiled Cys thiol redox in cells experiencing nutrient replete, carbon limited, and nitrogen limited cells when transitioning from light to dark irradiance. Nutrient deficient cultures had increased levels of Cys thiol reduction than nutrient replete samples, a phenomenon indicative of unbalanced electron transport and ATP synthesis [33]. Both studies yielded novel proteins undergoing redox dynamics while also confirming well known redox regulated proteins, and provide new clues for redox 'switches' that may be coupled directly to metabolic output. 


\section{Conclusion}

Human health, energy production, environmental remediation, and climate change all are tightly coupled to microbial physiology. As human microbiome research has already demonstrated, humans and the planet as a whole have a deep connection to microbes for nearly all aspects of life. However, the vast diversity of microbes, the plurality of microbial interactions, and the breadth of environments they reside places researchers at a point in which we have hardly scratched the surface of microbial discovery. Harnessing the utility of ABPP for continued microbial research will facilitate our understanding of protein functions, interactions, and regulation. Moving forward, the multimodal nature of ABPP enables the use of mass spectrometry-based proteomics, and also powerful microscopy and imaging techniques, such as fluorescence and electron microscopy. Taken together, microbial metabolism, community functions and assemblages, interactions, spatiotemporal dynamics, and response to perturbation can be untangled with increasing effectiveness.

\section{Acknowledgements}

We thank Richard D. Smith for his careful edits and insightful suggestions. Portions of this research were supported by the Department of Energy Office of Biological and Environmental Research Genome Sciences Program under the Pan-omics and Foundational Scientific Focus Area projects, and the DOE-BER Bioimaging Pilot Project program; additional resources supporting research described herein was provided via the Environmental Molecular Sciences Laboratory, a DOE-BER national scientific user facility at Pacific Northwest National Laboratory (PNNL) in Richland, Washington. PNNL is operated by Battelle for the DOE under contract DEAC05-76RLO-1830. 
1. Rostovtsev VV, Green LG, Fokin VV, Sharpless KB: A stepwise huisgen cycloaddition process: copper(I)-catalyzed regioselective "ligation" of azides and terminal alkynes. Angew Chem Int Ed Engl 2002, 41:2596-2599.

2. Speers AE, Cravatt BF: Profiling enzyme activities in vivo using click chemistry methods. Chem Biol 2004, 11:535-546.

3. Jeffery DA, Bogyo M: Chemical proteomics and its application to drug discovery. Curr Opin Biotechnol 2003, 14:87-95.

4. Kozarich JW: Activity-based proteomics: enzyme chemistry redux. Curr Opin Chem Biol 2003, 7:7883.

5. Chan EW, Chattopadhaya S, Panicker RC, Huang X, Yao SQ: Developing photoactive affinity probes for proteomic profiling: hydroxamate-based probes for metalloproteases. J Am Chem Soc 2004, 126:14435-14446.

6. Staub I, Sieber SA: Beta-lactams as selective chemical probes for the in vivo labeling of bacterial enzymes involved in cell wall biosynthesis, antibiotic resistance, and virulence. J Am Chem Soc 2008, 130:13400-13409.

7. Bottcher T, Sieber SA: Showdomycin as a versatile chemical tool for the detection of pathogenesisassociated enzymes in bacteria. J Am Chem Soc 2010, 132:6964-6972.

8. Eirich J, Orth R, Sieber SA: Unraveling the protein targets of vancomycin in living S. aureus and $E$. faecalis cells. J Am Chem Soc 2011, 133:12144-12153.

9. Kocaoglu O, Calvo RA, Sham LT, Cozy LM, Lanning BR, Francis S, Winkler ME, Kearns DB, Carlson EE: Selective penicillin-binding protein imaging probes reveal substructure in bacterial cell division. ACS Chem Biol 2012, 7:1746-1753.

*10. Battenberg OA, Yang Y, Verhelst SH, Sieber SA: Target profiling of 4-hydroxyderricin in S. aureus reveals seryl-tRNA synthetase binding and inhibition by covalent modification. Mol Biosyst 2013, 9:343-351.

A probe synthesized to mimic 4-hydroxyderricin, a naturally occuring chemical with antibiotic properties, and applied to Staphylococcus aureus. The 4-hydroxyderricin probe bound and inhibited seryl-tRNA synthetases (STS), enzymes crucial for the biosynthesis of proteins.

11. Liu Y, Patricelli MP, Cravatt BF: Activity-based protein profiling: the serine hydrolases. Proc Natl Acad Sci U S A 1999, 96:14694-14699.

12. Wiedner SD, Burnum KE, Pederson LM, Anderson LN, Fortuin S, Chauvigne-Hines LM, Shukla AK, Ansong $C$, Panisko EA, Smith RD, et al.: Multiplexed activity-based protein profiling of the human pathogen Aspergillus fumigatus reveals large functional changes upon exposure to human serum. J Biol Chem 2012, 287:33447-33459.

13. Wiedner SD, Ansong C, Webb-Robertson BJ, Pederson LM, Fortuin S, Hofstad BA, Shukla AK, Panisko EA, Smith RD, Wright AT: Disparate proteome responses of pathogenic and nonpathogenic aspergilli to human serum measured by activity-based protein profiling (ABPP). Mol Cell Proteomics 2013, 12:1791-1805.

14. Sherratt AR, Blais DR, Ghasriani H, Pezacki JP, Goto NK: Activity-based protein profiling of the Escherichia coli GIpG rhomboid protein delineates the catalytic core. Biochemistry 2012, 51:7794-7803.

15. Patricelli MP, Szardenings AK, Liyanage M, Nomanbhoy TK, Wu M, Weissig H, Aban A, Chun D, Tanner S, Kozarich JW: Functional interrogation of the kinome using nucleotide acyl phosphates. Biochemistry 2007, 46:350-358.

16. Ansong C, Ortega C, Payne SH, Haft DH, Chauvigne-Hines LM, Lewis MP, Ollodart AR, Purvine SO, Shukla AK, Fortuin $S$, et al.: Identification of widespread adenosine nucleotide binding in Mycobacterium tuberculosis. Chem Biol 2013, 20:123-133. 
17. Wolfe LM, Veeraraghavan U, Idicula-Thomas S, Schurer S, Wennerberg K, Reynolds R, Besra GS, Dobos KM: A chemical proteomics approach to profiling the ATP-binding proteome of Mycobacterium tuberculosis. Mol Cell Proteomics 2013, 12:1644-1660.

**18. Ortega C, Liao R, Anderson LN, Rustad T, Ollodart AR, Wright AT, Sherman DR, Grundner C: Mycobacterium tuberculosis Ser/Thr protein kinase B mediates an oxygen-dependent replication switch. PLOS Biol 2014, 12:e1001746.

ATP derived probes were applied to Mycobacterium tuberculosis in order to illuminate on the ATP-binding proteins that are active participants in the oxygen-dependent replication switch involved in latency.

19. Nishino M, Choy JW, Gushwa NN, Oses-Prieto JA, Koupparis K, Burlingame AL, Renslo AR, McKerrow $\mathrm{JH}$, Taunton J: Hypothemycin, a fungal natural product, identifies therapeutic targets in Trypanosoma brucei [corrected]. Elife 2013, 2:e00712.

20. Yestrepsky BD, Kretz CA, Xu Y, Holmes A, Sun H, Ginsburg D, Larsen SD: Development of tag-free photoprobes for studies aimed at identifying the target of novel Group A Streptococcus antivirulence agents. Bioorg Med Chem Lett 2014, 24:1538-1544.

21. Clarke KM, Mercer AC, La Clair JJ, Burkart MD: In vivo reporter labeling of proteins via metabolic delivery of coenzyme A analogues. J Am Chem Soc 2005, 127:11234-11235.

22. Meier JL, Mercer AC, Rivera H, Jr., Burkart MD: Synthesis and evaluation of bioorthogonal pantetheine analogues for in vivo protein modification. J Am Chem Soc 2006, 128:1217412184.

23. Mercer AC, Meier JL, Torpey JW, Burkart MD: In vivo modification of native carrier protein domains. Chembiochem 2009, 10:1091-1100.

*24. Chauvigne-Hines LM, Anderson LN, Weaver HM, Brown JN, Koech PK, Nicora CD, Hofstad BA, Smith RD, Wilkins MJ, Callister SJ, et al.: Suite of activity-based probes for cellulose-degrading enzymes. J Am Chem Soc 2012, 134:20521-20532.

A suite of novel activity based glycoside hydrolase targeting probes were synthesized and applied to Clostridium thermocellum. Proteomics provided a picture of the functional assembly of the cellulosome.

25. Anderson LN, Culley DE, Hofstad BA, Chauvigne-Hines LM, Zink EM, Purvine SO, Smith RD, Callister SJ, Magnuson JM, Wright AT: Activity-based protein profiling of secreted cellulolytic enzyme activity dynamics in Trichoderma reesei QM6a, NG14, and RUT-C30. Mol Biosyst 2013, 9:29923000.

26. Gygi SP, Rist B, Gerber SA, Turecek F, Gelb MH, Aebersold R: Quantitative analysis of complex protein mixtures using isotope-coded affinity tags. Nat Biotechnol 1999, 17:994-999.

27. Shiio Y, Aebersold R: Quantitative proteome analysis using isotope-coded affinity tags and mass spectrometry. Nat Protoc 2006, 1:139-145.

28. Weerapana E, Wang C, Simon GM, Richter F, Khare S, Dillon MB, Bachovchin DA, Mowen K, Baker D, Cravatt BF: Quantitative reactivity profiling predicts functional cysteines in proteomes. Nature 2010, 468:790-795.

**29. Deng X, Weerapana E, Ulanovskaya O, Sun F, Liang H, Ji Q, Ye Y, Fu Y, Zhou L, Li J, et al.: Proteomewide quantification and characterization of oxidation-sensitive cysteines in pathogenic bacteria. Cell Host Microbe 2013, 13:358-370.

Isotopic variants of iodoacetamide derived probes were used to identify and quantify $\mathrm{H}_{2} \mathrm{O}_{2}$ sensitive proteins in $P$. aeruginosa and $S$. aureus; LasR, ExaC, ArCA, and GAPDH were among the highlighted targets and were confirmed through mutation and activity assays.

30. Leichert LI, Jakob U: Protein thiol modifications visualized in vivo. PLoS Biol 2004, 2:e333. 
31. Leichert LI, Gehrke F, Gudiseva HV, Blackwell T, Ilbert M, Walker AK, Strahler JR, Andrews PC, Jakob $\mathrm{U}$ : Quantifying changes in the thiol redox proteome upon oxidative stress in vivo. Proc Natl Acad Sci U S A 2008, 105:8197-8202.

32. Sadler NC, Melnicki MR, Serres MH, Merkley ED, Chrisler WB, Hill EA, Romine MF, Kim S, Zink EM, Datta $S$, et al.: Live cell chemical profiling of temporal redox dynamics in a photoautotrophic cyanobacterium. ACS Chem Biol 2014, 9:291-300.

33. Ansong C, Sadler NC, Hill EA, Lewis MP, Zink EM, Smith RD, Beliaev AS, Konopka AE, Wright AT: Characterization of protein redox dynamics induced during light-to-dark transitions and nutrient limitation in cyanobacteria. Front Microbiol 2014, 5:325. 


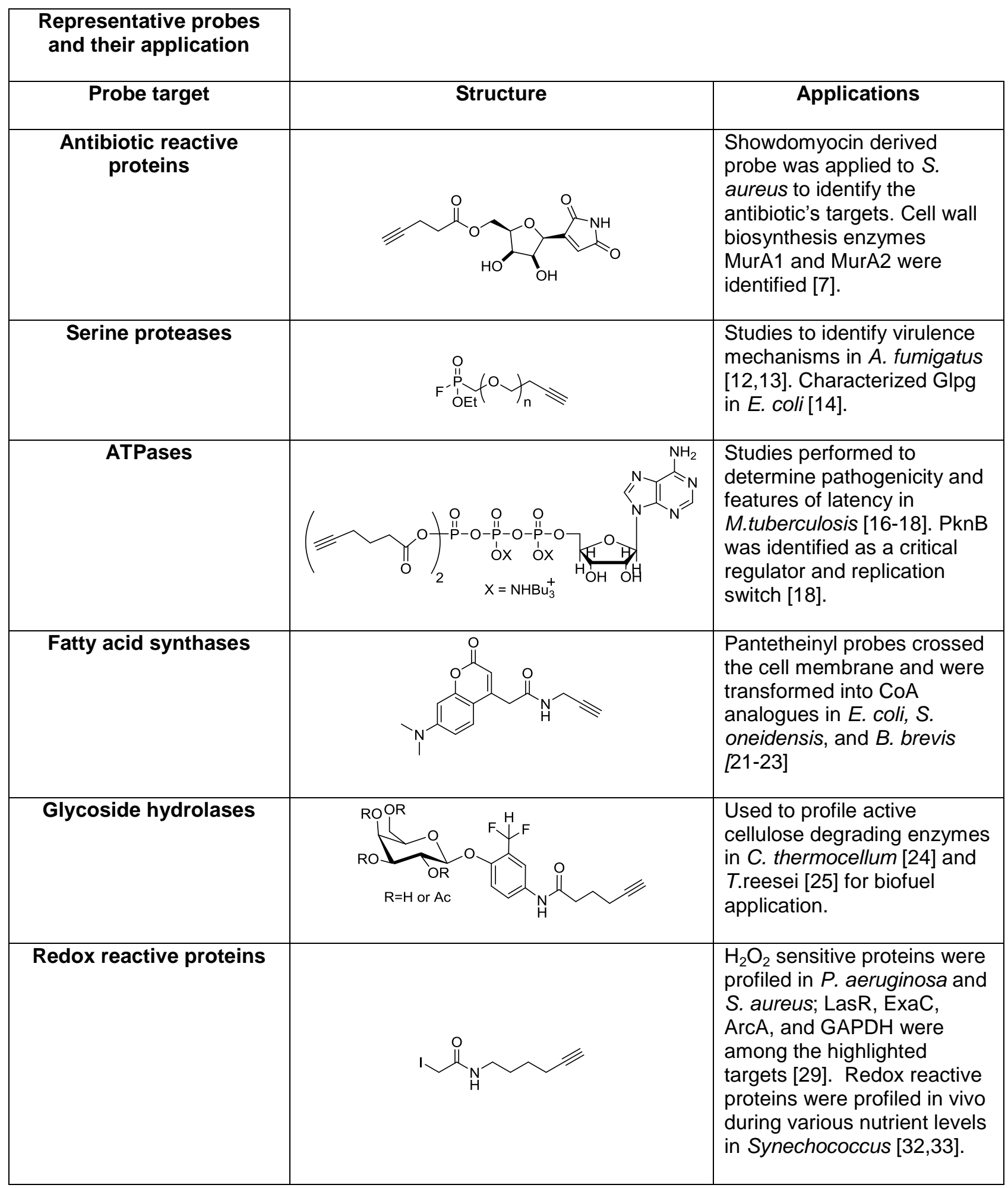


Figure 1. "Tagged" versus "Tag-Free" activity-based protein profiling. "Tagged" probes directly incorporate the reporter or enrichment moiety directly into the probe structure. "Tag-free" probes are enabled for use in [3+2] Huisgen-Sharpless cycloaddition reactions, delivering the reporter moiety postprotein labeling. Both variants can be used for subsequent SDS-PAGE, fluorescence imaging, or LC-MS proteomic analysis of probe targets. 


\section{Probe design and classification}

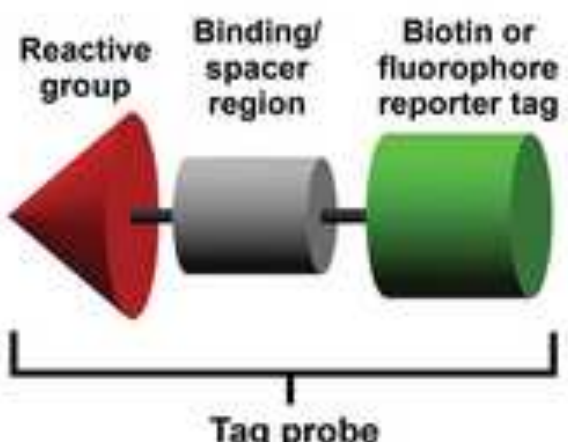

Tag probe

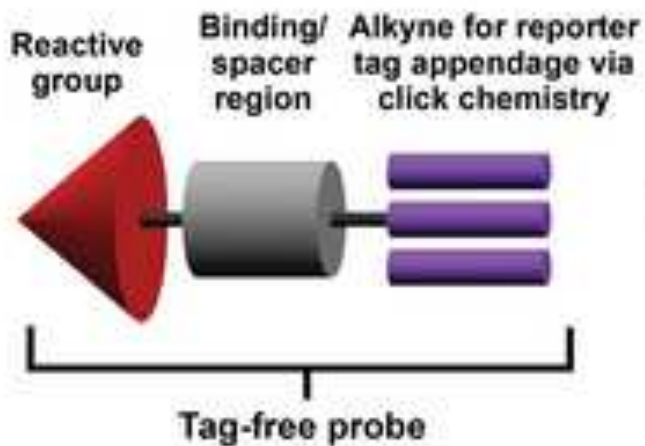

Tag-free probe
Biotin- $\mathrm{N}_{3}$ or

Fluor- $\mathrm{N}_{3}$
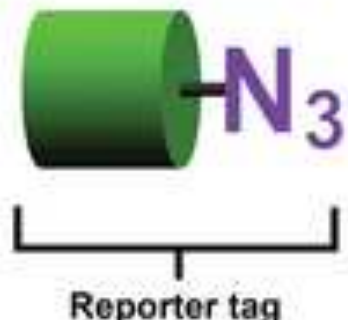

\section{Probe labeling of reactive proteins}

\section{Tagged ABPP}

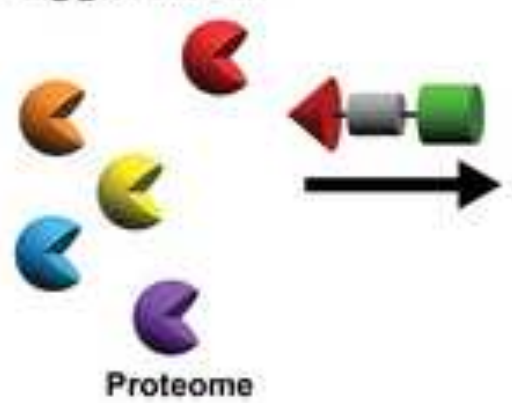

Tag-free ABPP

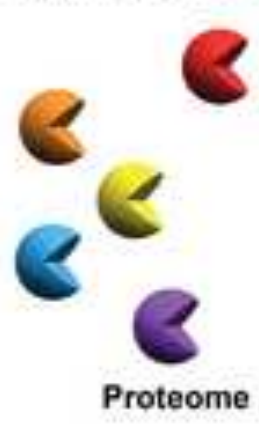

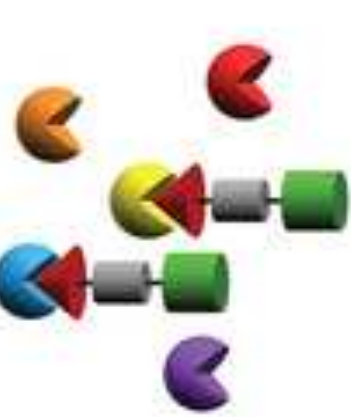

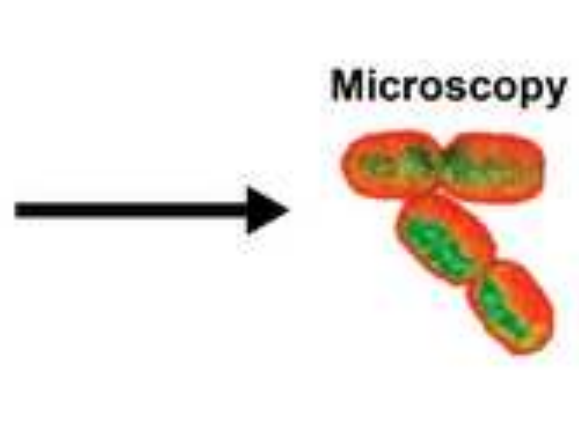

SDS

PAGE Spectrometry

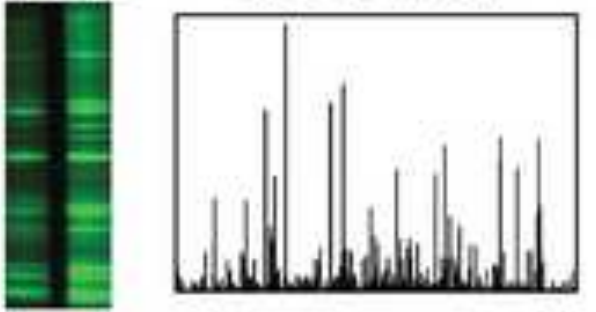

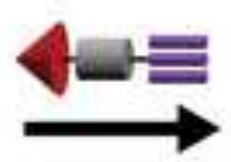

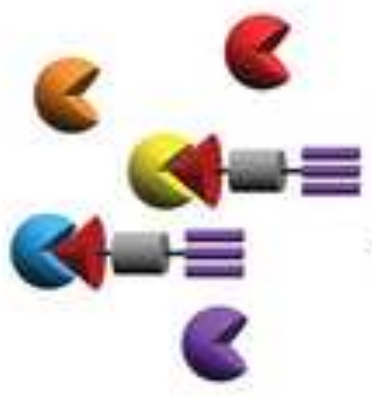

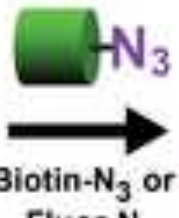

Fluor- $\mathrm{N}_{3}$
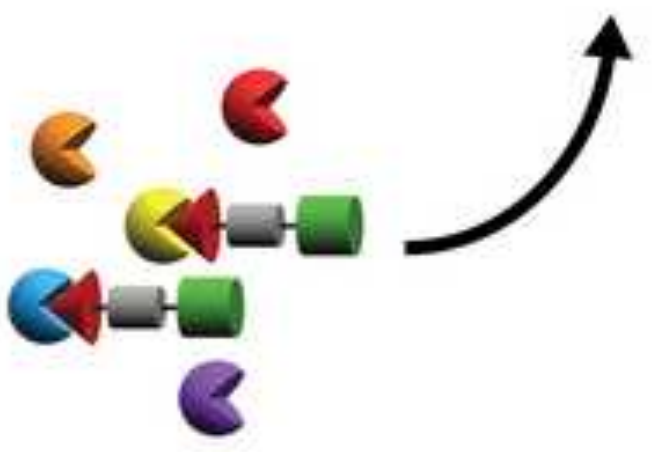